\title{
Effects of Pneumococcal Conjugate Vaccine on Genotypic Penicilín Resistance and Serotype Changes, Japan, 2010-2017
}

\author{
Kimiko Ubukata, Misako Takata, Miyuki Morozumi, Naoko Chiba, Takeaki Wajima, \\ Shigeo Hanada, Michi Shouji, Megumi Sakuma, Satoshi Iwata; \\ the Invasive Pneumococcal Diseases Surveillance Study Group
}

To clarify year-to-year changes in capsular serotypes, resistance genotypes, and multilocus sequence types of Streptococcus pneumoniae, we compared isolates collected from patients with invasive pneumococcal disease before and after introductions of 7 - and 13-valent pneumococcal conjugate vaccines (PCV7 and PVC13, respectively). From April 2010 through March 2017, we collected 2,856 isolates from children and adults throughout Japan. Proportions of PCV13 serotypes among children decreased from $89.0 \%$ in fiscal year 2010 to $12.1 \%$ in fiscal year 2016 and among adults from $74.1 \%$ to $36.2 \%$. Although nonvaccine serotypes increased after introduction of PCV13, genotypic penicillin resistance decreased from $54.3 \%$ in 2010 to $11.2 \%$ in 2016 among children and from $32.4 \%$ to $15.5 \%$ among adults. However, genotypic penicillin resistance emerged in 9 nonvaccine serotypes, but not 15A and 35B. Multilocus sequence typing suggested that resistant strains among nonvaccine serotypes may have evolved from clonal complexes 156 and 81 . A more broadly effective vaccine is needed.

A mong persons in all age groups, but particularly infants and elderly persons, Streptococcus pneumoniae remains a major cause of invasive pneumococcal disease (IPD) (e.g., pneumonia, meningitis, and sepsis), although generally effective antimicrobial agents are available (l). In the United States, 7-valent pneumococcal conjugate vaccine (PCV7) has been administered to children since 2000, resulting in both individual and herd immunity, with declines in pneumococcal infection among children and elderly persons (2-6). Unfortunately, introduction of PCV7

Author affiliations: Keio University School of Medicine, Tokyo, Japan (K. Ubukata, M. Takata, M. Morozumi, N. Chiba,

M. Sakuma, S. Iwata); Tokyo University of Pharmacy and Life Sciences, Tokyo (T. Wajima); Toranomon Hospital, Tokyo (S. Hanada); National Cancer Center Hospital, Tokyo (M. Shouji, S. Iwata)

DOI: https://doi.org/10.3201/eid2411.180326 was followed by an increase in serotype 19A showing penicillin resistance and often multidrug resistance (5-8). In 2010 , vaccination for children was upgraded to 13 -valent pneumococcal conjugate vaccine (PCV13), which covers 6 additional serotypes: $1,3,5,6 \mathrm{~A}, 7 \mathrm{~F}$, and 19A (9). Introduction of PCV13 contributed to decreases in $\operatorname{IPD}(10,11)$, pneumonia (including community-acquired pneumonia without bacteremia) $(12,13)$, and acute otitis media $(14-16)$ caused by $S$. pneumoniae belonging to vaccine serotypes, especially 6A and 19A. As an indirect effect of wide administration of PCVs to children, pneumococcal infections in adults have also decreased, representing herd immunity $(11,12,17-23)$.

Despite these benefits, in countries where PCV7 or PCV13 was introduced, proportions of disease preventable by PCVs gradually decreased because vaccine-serotype pneumococci were replaced by nonvaccine serotypes (NVTs). Increases in NVTs such as $6 \mathrm{C}, 15 \mathrm{~A} / \mathrm{B} / \mathrm{C}, 23 \mathrm{~A}$, and $35 \mathrm{~B}$ have been reported in the United States (24-28); 15A and 23B in Norway (18) and Germany (29); and 12F, 15A, 24F, and 35B in France (30).

In November 2010 in Japan, PCV7 vaccination use among children $<5$ years of age was introduced voluntarily by the Provisional Special Fund for the Urgent Promotion of Vaccination. In April 2013, PCV7 was officially incorporated into the vaccination program as public administration; in November of that year, PCV7 was replaced by PCV13. Promotion of PCV7 vaccination for children rapidly halved the number of IPD cases caused by vaccine-serotype pneumococci among children (31) and also produced a herd effect benefiting elderly persons (32). After PCV7 introduction, however, among persons of all ages, IPD caused by non-PCV7 serotypes such as 19A, 15A, 15B, 15C, 22F, and $24 \mathrm{~F}$ showed relative increases in 2013. In November 2014, the Japanese Ministry of Health, Labour and Welfare began promoting vaccination of adults $\geq 65$ years of age with 23 -valent pneumococcal polysaccharide vaccine 
(PPSV23). In this study, we aimed to clarify year-to-year changes in capsular serotypes, genotypes of penicillin and macrolide resistance, and diversity of sequence types (STs) in all pneumococcal isolates collected throughout Japan during April 2010-March 2017.

\section{Methods}

\section{Patients and Pneumococcal Strains}

We included all specimens from patients of any age with IPD. Pneumococcal isolates from normally sterile clinical samples were collected from clinical laboratories at 341 hospitals participating in this IPD surveillance study. Each hospital had a microbiology laboratory as described previously (31), and participating hospitals were distributed nearly uniformly throughout Japan. These hospitals took part in the surveillance project after written permission was granted by the laboratory director or hospital director. This study was approved by the Keio University School of Medicine Ethics Committee (approval no. 20140432).

A total of 2,856 pneumococcal strains were collected from April 2010 through March 2017 (online Technical Appendix Figure 1, https://wwwnc.cdc.gov/EID/ article/24/11/18-0326-Techapp1.pdf). The first surveillance interval, April 2010-March 2011 (designated 2010), represented the pre-PCV7 period. The second surveillance interval, April 2011-March 2014 (designated 2011-2013, the PCV7 period), showed effects of PCV7 vaccination for children $<5$ years of age. The third surveillance interval, April 2014-March 2017 (designated 2014-2016, the PCV13 period), reflected PCV13 vaccination for children $<5$ years of age.

During the pre-PCV7 period, the rate of voluntary PCV7 vaccination among children in Japan was $<10 \%$. The PCV7 period corresponded to the Urgent Promotion of PCV7, a vaccination incentive program for children. The PCV7 vaccination rate throughout Japan was estimated at $50 \%-60 \%$ in $2011,80 \%-90 \%$ in 2012 , and $>95 \%$ in 2013. During the PCV13 period, corresponding to substitution of PCV13 for routine vaccination, coverage remained $>95 \%$. In elderly persons ( $\geq 65$ years of age), the rate of vaccination with PPSV23, starting in 2014, has remained at $\approx 54 \%$ as of 2017 (Vaccine Medical Affairs of Merck Sharp and Dohme K.K., Tokyo, Japan, pers. comm., 2017 Apr 1).

Pneumococcal isolates were sent promptly from each clinical laboratory to the Department of Infectious Diseases, Keio University School of Medicine (Tokyo, Japan), accompanied by a survey form completed by the attending physician. In compliance with ethics guidelines for epidemiology in Japan, patients were not identified.

\section{Serotype and Resistance Genotype}

We determined serotypes by using the capsular quellung test with antiserum purchased from Statens Serum
Institute (Copenhagen, Denmark). Alterations in 3 penicillin-binding protein genes that mediate $\beta$-lactam resistance in $S$. pneumoniae ( $p b p 1 a, p b p 2 x$, and $p b p 2 b$ ) were identified by real-time PCR as described previously (33). The $m e f(\mathrm{~A})$ and $\operatorname{erm}(\mathrm{B})$ genes, which mediate macrolide resistance, were also identified by real-time PCR (33). Quinolone resistance was analyzed by sequencing the quinolone resistance-determining region in the genes gyrA, gyrB, $\operatorname{par} C$, and parE in strains showing MICs of levofloxacin exceeding $4 \mu \mathrm{g} / \mathrm{mL}$.

Genotypes (g) based on gene analysis were represented as follows: penicillin-susceptible $S$. pneumoniae (gPSSP), possessing 3 normal $p b p$ genes; penicillin-intermediate S. pneumoniae (gPISP), subclassified as gPISP $(p b p 2 x)$, gPISP $(p b p 2 b)$, gPISP $(p b p 1 a+p b p 2 x)$, gPISP $(p b p 1 a+p b p 2 b)$, or gPISP $(p b p 2 x+p b p 2 b)$; and penicillinresistant $S$. pneumoniae (gPRSP), which possessed 3 abnormal $p b p$ genes $(31,33)$. Serotype and resistance genotype results were promptly reported to laboratory staff at each referring hospital.

\section{Susceptibility Testing}

For all isolates, we redetermined the MICs of 6 antimicrobial agents by using agar-dilution methods with reference strains R6 and ATCC49619(34). The agents tested were penicillin, ampicillin, cefotaxime, meropenem, vancomycin, and levofloxacin.

\section{Multilocus Sequence Typing}

We performed multilocus sequence typing (MLST) analysis for all 2,849 isolates that could be cultured. Primers used for MLST were based on sequences listed at https://pubmlst. org/spneumoniae/. Clusters of related STs were analyzed by using eBURST version 3 (http://eburst.mlst.net/).

\section{Statistical Analyses}

For statistical analyses, we used Ekuseru-Toukei 2015 software (Social Survey Research Information, Tokyo, Japan) and R software 3.5.0 (R Foundation of Computational Statistics, Vienna, Austria). We used the $\chi^{2}$ and Fisher exact tests as appropriate. We considered $\mathrm{p}<0.05$ to indicate statistical significance.

\section{Results}

\section{Relationships between IPD Type and Patient Age}

Relationships between IPD type and patient age are shown in Table 1. IPD types were classified into 4 categories: pneumonia with bacteremia (41.9\%), including empyema and pleuritis; bacteremia with unknown focus $(37.0 \%)$; meningitis (15.4\%); and others (5.6\%), including endocarditis, necrotizing fasciitis, cellulitis, arthritis, and spondylitis. Pneumonia with bacteremia was most common among adults, especially 
Table 1. Invasive pneumococcal disease in all patients, by age group, Japan, April 2010-March 2017

\begin{tabular}{|c|c|c|c|c|c|c|c|c|c|c|}
\hline \multirow[b]{2}{*}{ Disease } & \multirow{2}{*}{$\begin{array}{c}\text { Total, } \\
\text { no. }(\%), \\
\text { n=2,856 }\end{array}$} & \multicolumn{8}{|c|}{ Age, y, no. (\%) } & \multirow[b]{2}{*}{$p$ value } \\
\hline & & $\begin{aligned} & \leq 2 \\
\mathrm{n} & =731\end{aligned}$ & $\begin{array}{c}3-5 \\
n=181\end{array}$ & $\begin{array}{l}6-17 \\
\mathrm{n}=94\end{array}$ & $\begin{array}{l}18-49 \\
n=201\end{array}$ & $\begin{array}{l}50-64, \\
n=387\end{array}$ & $\begin{array}{l}65-74 \\
n=530\end{array}$ & $\begin{array}{l}75-84 \\
\mathrm{n}=457\end{array}$ & $\begin{aligned} & \geq 85 \\
& \mathrm{n}=275\end{aligned}$ & \\
\hline $\begin{array}{l}\text { Pneumonia with } \\
\text { bacteremia* }^{*}\end{array}$ & $\begin{array}{l}1,198 \\
(41.9)\end{array}$ & $130(17.8)$ & $35(19.3)$ & $22(23.4)$ & $83(41.3)$ & $167(43.2)$ & $261(49.2)$ & $300(65.6)$ & $200(72.7)$ & $<0.001$ \\
\hline $\begin{array}{l}\text { Bacteremia with } \\
\text { focus unknown }\end{array}$ & $\begin{array}{l}1,058 \\
(37.0)\end{array}$ & $455(62.2)$ & $116(64.1)$ & 33 (35.1) & $46(22.9)$ & $104(26.9)$ & $158(29.8)$ & $92(20.1)$ & 54 (19.6) & $<0.001$ \\
\hline Meningitis & $440(15.4)$ & 109 (14.9) & $22(12.2)$ & $34(36.2)$ & $56(27.9)$ & $80(20.7)$ & 79 (14.9) & $43(9.4)$ & $17(6.2)$ & $<0.001$ \\
\hline Other† & $160(5.6)$ & $37(5.1)$ & $8(4.4)$ & $5(5.3)$ & $16(8.0)$ & $36(9.3)$ & $32(6.0)$ & $22(4.8)$ & $4(1.5)$ & 0.002 \\
\hline
\end{tabular}

those $\geq 75$ years of age; however, among children $<5$ years of age, bacteremia with unknown focus was most common $(p<0.001$ for each). Meningitis and other IPDs were represented in higher proportions among persons 6-64 years of age $(p<0.001)$ than among those in other age groups $(p=0.002)$.

\section{Changes in Serotypes}

Figure 1 shows yearly changes in pneumococcal capsular serotypes among children and adults. Pneumococcal capsular serotypes were classified into 4 groups: PCV7 serotypes (4, 6B, 9V, 14, 18C, 19F, and 23F) (PCV7); PCV13 serotypes not included in PCV7 $(1,3,5,6 \mathrm{~A}, 7 \mathrm{~F}$, and 19A) (PCV13-nonPCV7); PPSV23 serotypes not included in PCV13 $(2,8,9 \mathrm{~N}, 10 \mathrm{~A}, 11 \mathrm{~A}, 12 \mathrm{~F}, 15 \mathrm{~B}, 17 \mathrm{~F}, 20,22 \mathrm{~F}$, and 33F) (PPSV23-nonPCV13); and NVTs not including serotypes in PPSV23 and not including 6A. Among children, the proportion of PCV7 serotypes that accounted for $73.3 \%$ of serotype strains isolated from IPD patients during the pre-PCV7 period decreased rapidly to $7.4 \%$ in 2013 after PCV7 introduction (Figure 1). In contrast, in 2013, PCV13-nonPCV7 serotypes increased from 15.7\% to $25.9 \%$, PPSV23-nonPCV13 serotypes increased from $3.0 \%$ to $18.5 \%$, and NVT serotypes increased from $8.0 \%$ to $48.1 \%$. During 2014, after PCV7 was replaced with PCV13, the proportion of PCV13-nonPCV7 serotypes decreased by approximately half to $11.1 \%$ in 2016 , while
PPSV23-nonPCV13 serotypes increased to $40.4 \%$, in contrast to the PCV7 period.

Among adults, the proportions of PCV7 serotypes, which accounted for $43.6 \%$ of isolates during the pre-PCV7 period, decreased to $11.7 \%$ in 2013, when children were vaccinated with PCV7. However, proportions of the PPSV23nonPCV13 doubled from $12.4 \%$ to $25.4 \%$ and NVTs doubled from $13.5 \%$ to $28.9 \%$. PCV13-nonPCV7 serotypes decreased slightly after replacement by PCV13 in 2014, but PPSV23-nonPCV13 serotypes continued to increase.

\section{Serotype Changes during the Pre-PCV7, PCV7, and PCV13 Periods}

Changes in serotypes of pneumococcal isolates collected between the pre-PCV7, PCV7, and PCV13 periods are shown in Table 2 for children and in Table 3 for adults. Among children, proportions of PCV7 serotypes decreased rapidly from $73.3 \%$ to $30.3 \%$ during the PCV7 period and decreased further to $2.3 \%$ during the PCV13 period $(\mathrm{p}<0.001)$. Among PCV13-nonPCV7 serotypes, serotype 19A apparently increased during the PCV7 period, but later it decreased significantly during the PCV13 period. PCV13-nonPCV7 serotypes decreased from $21.8 \%$ during the PCV7 period to $14.9 \%$ during the PCV13 period $(\mathrm{p}=0.031)$. Although serotypes 1 and $7 \mathrm{~F}$ showed relative increases during the PCV13 period, most were isolated from patients $\geq 3$ years of
Figure 1. Yearly changes in pneumococcal serotypes of isolates from A) 1,006 children and $B) 1,850$ adults with invasive pneumococcal disease in Japan, April 2010-March 2017. Specific percentages are indicated at points along data lines. Fiscal years extend from April 1 through March 31 of the following year. PCV13-nonPCV7 covers 6 serotypes (1, 3, 5, 6A, 7F, and 19A). PPSV23-nonPCV13 covers 11 serotypes $(2,8,9 \mathrm{~N}, 10 \mathrm{~A}$, $11 \mathrm{~A}, 12 \mathrm{~F}, 15 \mathrm{~B}, 17 \mathrm{~F}, 20,22 \mathrm{~F}$, and $33 \mathrm{~F})$, but $2,9 \mathrm{~N}$, and $17 \mathrm{~F}$ were not isolated in this study. NVTs represent other serotypes not included in PPSV23 and 6A. NVT, nonvaccine serotype; PCV7, 7-valent pneumococcal conjugate vaccine; PCV13, 13-valent pneumococcal conjugate vaccine; PPSV23, 23-valent pneumococcal polysaccharide vaccine.

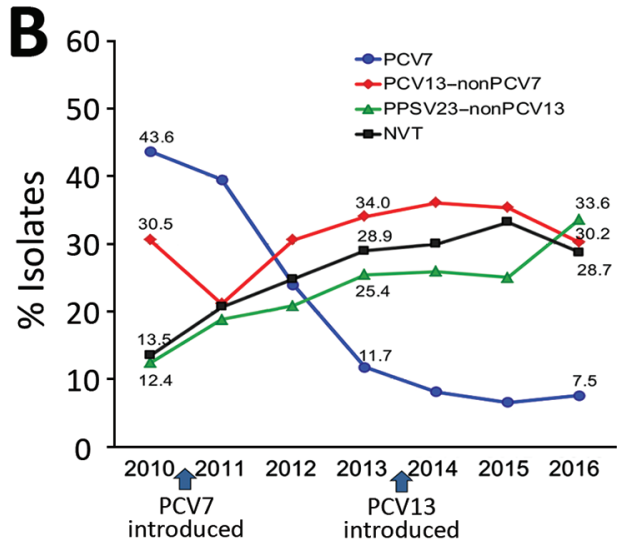


Table 2. Distribution of pneumococcal serotypes among children before PCV7 and after introduction of PCV7 and PCV13, Japan, April 2010-March 2017*

\begin{tabular}{|c|c|c|c|c|}
\hline \multirow[b]{2}{*}{ Serotype } & \multicolumn{3}{|c|}{ No. (\%) } & \multirow[b]{2}{*}{$\mathrm{p}$ value } \\
\hline & $\begin{array}{c}\text { Pre-PCV7 period, 2010, } \\
n=300\end{array}$ & $\begin{array}{c}\text { PCV7 period 2011-2013, } \\
n=357\end{array}$ & $\begin{array}{l}\text { PCV13 period, 2014-2016, } \\
n=349\end{array}$ & \\
\hline \multicolumn{5}{|l|}{ PCV7 } \\
\hline 4 & $7(2.3)$ & $6(1.7)$ & 0 & 0.007 \\
\hline $6 \mathrm{~B}$ & $83(27.7)$ & $49(13.7)$ & $2(0.6)$ & $<0.001$ \\
\hline $9 \mathrm{~V}$ & $9(3.0)$ & $2(0.6)$ & $1(0.3)$ & 0.005 \\
\hline 14 & $34(11.3)$ & $9(2.5)$ & 0 & $<0.001$ \\
\hline $18 \mathrm{C}$ & $4(1.3)$ & $4(1.1)$ & $1(0.3)$ & 0.336 \\
\hline $19 \mathrm{~F}$ & $40(13.3)$ & $13(3.6)$ & $2(0.6)$ & $<0.001$ \\
\hline $23 \mathrm{~F}$ & $43(14.3)$ & $25(7.0)$ & $2(0.6)$ & $<0.001$ \\
\hline Subtotal & $220(73.3)$ & $108(30.3)$ & $8(2.3)$ & $<0.001$ \\
\hline \multicolumn{5}{|c|}{ PCV13-nonPCV7 } \\
\hline 1 & 0 & $5(1.4)$ & $13(3.7)$ & $<0.001$ \\
\hline 3 & $4(1.3)$ & $7(2.0)$ & $4(1.1)$ & 0.724 \\
\hline 5 & 0 & 0 & 0 & NA \\
\hline $6 \mathrm{~A}$ & $15(5.0)$ & $6(1.7)$ & $2(0.6)$ & 0.001 \\
\hline $7 \mathrm{~F}$ & $1(0.3)$ & $2(0.6)$ & $6(1.7)$ & 0.153 \\
\hline $19 \mathrm{~A}$ & $27(9.0)$ & $58(16.2)$ & $27(7.7)$ & 0.001 \\
\hline Subtotal & $47(15.7)$ & $78(21.8)$ & $52(14.9)$ & 0.031 \\
\hline \multicolumn{5}{|c|}{ PPSV23-nonPCV13§ } \\
\hline 8 & 0 & 0 & 0 & NA \\
\hline $10 \mathrm{~A}$ & $2(0.7)$ & $5(1.4)$ & $18(5.2)$ & $<0.001$ \\
\hline $11 \mathrm{~A}$ & $2(0.7)$ & $1(0.3)$ & $4(1.1)$ & 0.356 \\
\hline $12 \mathrm{~F}$ & $1(0.3)$ & $1(0.3)$ & $33(9.5)$ & $<0.001$ \\
\hline $15 B$ & 0 & $15(4.2)$ & $26(7.4)$ & $<0.001$ \\
\hline 20 & 0 & $1(0.3)$ & 0 & NA \\
\hline $22 \mathrm{~F}$ & $3(1.0)$ & $16(4.5)$ & $19(5.4)$ & 0.003 \\
\hline $33 \mathrm{~F}$ & $1(0.3)$ & $7(2.0)$ & $17(4.9)$ & 0.001 \\
\hline Subtotal & $9(3.0)$ & $46(12.9)$ & $117(33.5)$ & $<0.001$ \\
\hline \multicolumn{5}{|l|}{ NVT } \\
\hline $6 \mathrm{C}$ & $9(3.0)$ & $20(5.6)$ & $11(3.2)$ & 0.159 \\
\hline $15 \mathrm{~A}$ & $2(0.7)$ & $25(7.0)$ & $36(10.3)$ & $<0.001$ \\
\hline $15 \mathrm{C}$ & $1(0.3)$ & $17(4.8)$ & $16(4.6)$ & $<0.001$ \\
\hline $23 \mathrm{~A}$ & $4(1.3)$ & $13(3.6)$ & $10(2.9)$ & 0.176 \\
\hline $24 \mathrm{~F}$ & $1(0.3)$ & $20(5.6)$ & 52 (14.9) & $<0.001$ \\
\hline $24 B$ & $2(0.7)$ & 4 (1.1) & $12(3.4)$ & 0.197 \\
\hline 34 & $1(0.3)$ & $6(1.7)$ & $7(2.0)$ & 0.126 \\
\hline $35 B$ & $1(0.3)$ & $10(2.8)$ & $11(3.2)$ & 0.016 \\
\hline 38 & $1(0.3)$ & $8(2.2)$ & $7(2.0)$ & 0.072 \\
\hline Otherף & $2(0.7)$ & $2(0.6)$ & $9(2.6)$ & 0.041 \\
\hline Subtotal & $24(8.0)$ & $125(35.0)$ & 171 (49.0)\# & $<0.001$ \\
\hline \multicolumn{5}{|c|}{$\begin{array}{l}\text { *Years run from April } 1 \text { through March } 31 \text { of the following year. NA, not applicable; NVT, nonvacc } \\
\text { vaccine; PCV13, 13-valent pneumococcal conjugate vaccine; PPSV23, } 23 \text {-valent pneumococcal } \\
\text { tp values compare the } 3 \text { surveillance periods; boldface indicates significant increase. } \\
\text { †Serotypes added to PCV7. } \\
\text { §Serotypes contained in PPSV23 but not PCV13. } \\
\text { TIncludes 7C }(n=2), 16 F(n=2), 21(n=2), 23 B(n=3), 28 A(n=1), 37(n=1) \text {, and } 31(n=2) \text {. } \\
\text { \#One strain identified as nontypeable was excluded from the table. }\end{array}$} \\
\hline
\end{tabular}

age who had received PCV7 or a single dose of PCV13. To the contrary, proportions of PPSV23-nonPCV13 serotypes and NVTs increased significantly between the pre-PCV7 period and the PCV7 period, continuing to increase up to the PCV13 period ( $\mathrm{p}<0.001$ for each). In particular, 9 serotypes $(10 \mathrm{~A}, 12 \mathrm{~F}, 15 \mathrm{~A}, 15 \mathrm{~B}, 15 \mathrm{C}, 22 \mathrm{~F}, 24 \mathrm{~F}, 33 \mathrm{~F}$, and $35 \mathrm{~B})$ increased significantly after introduction of $\mathrm{PCV} 7$ and PCV13.

Among adults, proportions of PCV7 serotypes decreased sharply, from $43.6 \%$ during the pre-PCV7 period to $24.2 \%$ during the PCV7 period and $7.3 \%$ during the PCV13 period, particularly for serotypes $4,6 \mathrm{~B}, 9 \mathrm{~V}, 14$, $19 \mathrm{~F}$, and 23F. PCV13-nonPCV7 serotypes increased in serotypes $7 \mathrm{~F}$ and $19 \mathrm{~A}$, whereas $6 \mathrm{~A}$ showed a significant decrease because of cross-immunity with 6B (Table 3 ). PPSV23-nonPCV13 serotypes and NVTs increased respectively from $12.4 \%$ and $13.5 \%$ during the pre-PCV7 period to $21.9 \%$ and $24.9 \%$ during the PCV7 period and further to $27.8 \%$ and $30.9 \%$ during the PCV13 period $(\mathrm{p}<0.001$ for each). In particular, significant increases were noted for serotypes $12 \mathrm{~F}, 15 \mathrm{C}, 22 \mathrm{~F}, 23 \mathrm{~A}, 24 \mathrm{~F}$, and 35B. Tendencies to increase did not attain significance for serotypes $11 \mathrm{~A}$ and $15 \mathrm{~A}$.

\section{Changes in Penicillin and Other Resistance Genotypes}

Figure 2 shows yearly changes of penicillin resistance genotypes among children and adults. Changes are shown from 
Table 3. Distribution of pneumococcal serotypes in adults before PCV7 and after introduction of PCV7 and PCV13 administration to children, Japan, April 2010-March 2017*

\begin{tabular}{|c|c|c|c|c|}
\hline \multirow[b]{2}{*}{ Serotype } & \multicolumn{3}{|c|}{ No. (\%) } & \multirow[b]{2}{*}{$\mathrm{p}$ value } \\
\hline & $\begin{array}{l}\text { Pre-PCV7 period, 2010, } \\
n=275\end{array}$ & $\begin{array}{l}\text { PCV7 period, 2011-2013, } \\
n=695\end{array}$ & $\begin{array}{l}\text { PCV13 period, 2014-2016, } \\
n=880\end{array}$ & \\
\hline \multicolumn{5}{|l|}{ PCV7 } \\
\hline 4 & $14(5.1)$ & $27(3.9)$ & $4(0.5)$ & $<0.001$ \\
\hline $6 B$ & $42(15.3)$ & $39(5.6)$ & $22(2.5)$ & $<0.001$ \\
\hline $9 \mathrm{~V}$ & $7(2.5)$ & $7(1.0)^{\prime}$ & $6(0.7)^{\prime}$ & 0.042 \\
\hline 14 & $21(7.6)$ & $41(5.9)$ & $6(0.7)$ & $<0.001$ \\
\hline $18 \mathrm{C}$ & $1(0.4)$ & $3(0.4)$ & $2(0.2)$ & 0.642 \\
\hline $19 \mathrm{~F}$ & $14(5.1)$ & $23(3.3)$ & $15(1.7)$ & 0.007 \\
\hline $23 \mathrm{~F}$ & $21(7.6)$ & $28(4.0)$ & $9(1.0)$ & $<0.001$ \\
\hline Subtotal & $120(43.6)$ & $168(24.2)$ & $64(7.3)$ & $<0.001$ \\
\hline \multicolumn{5}{|c|}{ PCV13-nonPCV7 } \\
\hline 1 & $1(0.4)$ & $4(0.6)$ & $13(1.5)$ & 0.145 \\
\hline 3 & $45(16.4)$ & $110(15.8)$ & $145(16.5)$ & 0.939 \\
\hline 5 & 0 & $1(0.1)$ & 0 & NA \\
\hline $6 \mathrm{~A}$ & $11(4.0)$ & $16(2.3)$ & $9(1.0)$ & 0.006 \\
\hline $7 F$ & $9(3.3)$ & $9(1.3)$ & $33(3.8)$ & 0.006 \\
\hline $19 \mathrm{~A}$ & $18(6.5)$ & $61(8.8)$ & 99 (11.3) & 0.045 \\
\hline Subtotal & $84(30.5)$ & $201(28.9)$ & $299(34.0)$ & 0.093 \\
\hline \multicolumn{5}{|c|}{ PPSV23-nonPCV13§ } \\
\hline 8 & 0 & $2(0.3)$ & 0 & NA \\
\hline $10 \mathrm{~A}$ & $10(3.6)$ & $34(4.9)$ & $54(6.1)$ & 0.244 \\
\hline $11 \mathrm{~A}$ & $3(1.1)$ & $23(3.3)$ & $34(3.9)$ & 0.058 \\
\hline $12 \mathrm{~F}$ & $5(1.8)$ & $5(0.7)$ & $63(7.2)$ & $<0.001$ \\
\hline $15 B$ & $3(1.1)$ & $14(2.0)$ & $10(1.1)$ & 0.356 \\
\hline 20 & $1(0.4)$ & $7(1.0)$ & $14(1.6)$ & 0.261 \\
\hline $22 \mathrm{~F}$ & $10(3.6)$ & $63(9.1)$ & $59(6.7)$ & 0.008 \\
\hline $33 \mathrm{~F}$ & $2(0.7)$ & $4(0.6)$ & $11(1.3)$ & 0.352 \\
\hline Subtotal & $34(12.4)$ & $152(21.9)$ & $245(27.8)$ & $<0.001$ \\
\hline \multicolumn{5}{|l|}{ NVT } \\
\hline $6 \mathrm{C}$ & $13(4.7)$ & $49(7.1)$ & $52(5.9)$ & 0.400 \\
\hline $15 \mathrm{~A}$ & $6(2.2)$ & $28(4.0)$ & $47(5.3)$ & 0.068 \\
\hline $15 \mathrm{C}$ & 0 & $12(1.7)$ & $7(0.8)$ & 0.034 \\
\hline $23 \mathrm{~A}$ & $2(0.7)$ & $33(4.7)$ & $50(5.7)$ & $<0.001$ \\
\hline $24 \mathrm{~F}$ & 0 & $11(1.6)$ & $16(1.8)$ & 0.049 \\
\hline 34 & $1(0.4)$ & $5(0.7)$ & $12(1.4)$ & 0.301 \\
\hline $35 \mathrm{~B}$ & $7(2.5)$ & $22(3.2)$ & $55(6.3)$ & 0.004 \\
\hline 38 & $3(1.1)$ & $7(1.0)$ & $11(1.3)$ & 0.955 \\
\hline Other & $5(1.8)$ & $6(0.9)$ & $22(2.5)$ & 0.042 \\
\hline Subtotal & $37(13.5)$ & 173 (24.9)\# & $272(30.9)$ & $<0.001$ \\
\hline \multicolumn{5}{|c|}{$\begin{array}{l}\text { "Years run April 1- March } 31 \text { of the following year. NA, not applicable; NVT, nonvaccine serotype; PCV7, 7-valent pneum } \\
\text { 13-valent pneumococcal conjugate vaccine; PPSV23, 23-valent pneumococcal polysaccharide vaccine. } \\
\text { tp values compare the } 3 \text { surveillance periods; boldface indicates significant increase. } \\
\text { fSerotypes added to PCV7. } \\
\text { §Serotypes contained in PPSV23 but not PCV13. } \\
\text { TIncludes } 6 D(n=2), 7 C(n=8), 13(n=1), 16 F(n=6), 18 B(n=1), 23 B(n=5), 31(n=3) \text {, and } 37(n=7) \text {. } \\
\text { \#One strain identified as nontypeable was excluded from the table. }\end{array}$} \\
\hline
\end{tabular}

the pre-PCV7 period to the PCV7 period and further to the PCV13 period.

Among children, the proportion of gPRSP declined sharply from $54.3 \%$ in 2010 during the pre-PCV7 period to $20.4 \%$ in 2013 during the PCV7 period; gPSSP and gPISP (pbp2x) increased (Figure 2). In 2016 during the PCV13 period, proportions of gPRSP and gPISP $(p b p 1 a+2 x)$ further declined to $11.2 \%$ and $6.1 \%$, respectively. However, gPISP ( $p b p 2 b)$ rapidly increased.

Among isolates from adults during the pre-PCV7 period, gPISP ( $p b p 2 x)$ was most common (34.9\%), followed by gPRSP (32.4\%) (Figure 2). gPSSP accounted for only $15.3 \%$. Similar to the trend for children during the PCV7 and PCV13 periods, gPRSP among adults continually decreased to $15.5 \%$ in 2016. However, also in 2016, gPISP (pbp $2 b$ ) among adults increased to $8.3 \%$, similar to the trend among children.

During the surveillance periods, macrolide-resistant isolates possessing mef(A) or erm(B) genes remained consistently high. Among children, proportions were $93.8 \%$ in 2010 and $91.8 \%$ in 2016; among adults, proportions were $87.2 \%$ in 2010 and $89.8 \%$ in 2016 . Prevalence of resistance genes was $59.8 \%$ for the erm (B) gene mediating high macrolide resistance, $19.6 \%$ for the mef(A) gene mediating intermediate resistance, and $11.6 \%$ for both erm(B) and mef(A) genes (data not shown).

Isolates with mutations in both $g y r A$ and $\operatorname{parC}$ genes, which are involved in resistance to quinolones, especially 
Figure 2. Yearly changes in genotypic penicillin resistance in isolates from A) 1,006 children and $B) 1,850$ adults with invasive pneumococcal disease in Japan, April 2010March 2017. Fiscal years extend from April 1 through March 31 of the following year. Genotypes based on abnormal $p b p 1 a, p b p 2 x$, and $p b p 2 b$ genes were identified by real-time PCR and are represented as gPRSP $(1 a+2 x+2 b)$, gPISP $(1 a+2 x)$, gPISP $(1 a+2 b), g P I S P$ $(2 x+2 b)$, gPISP $(2 x)$, gPISP $(2 b)$, and gPSSP. g, genotype; PCV7, 7-valent pneumococcal conjugate vaccine; PCV13, 13-valent pneumococcal conjugate vaccine; PISP, penicillin-intermediate

Streptococcus pneumoniae; PRSP, penicillin-resistant S. pneumoniae; PSSP, penicillin-susceptible S. pneumoniae.
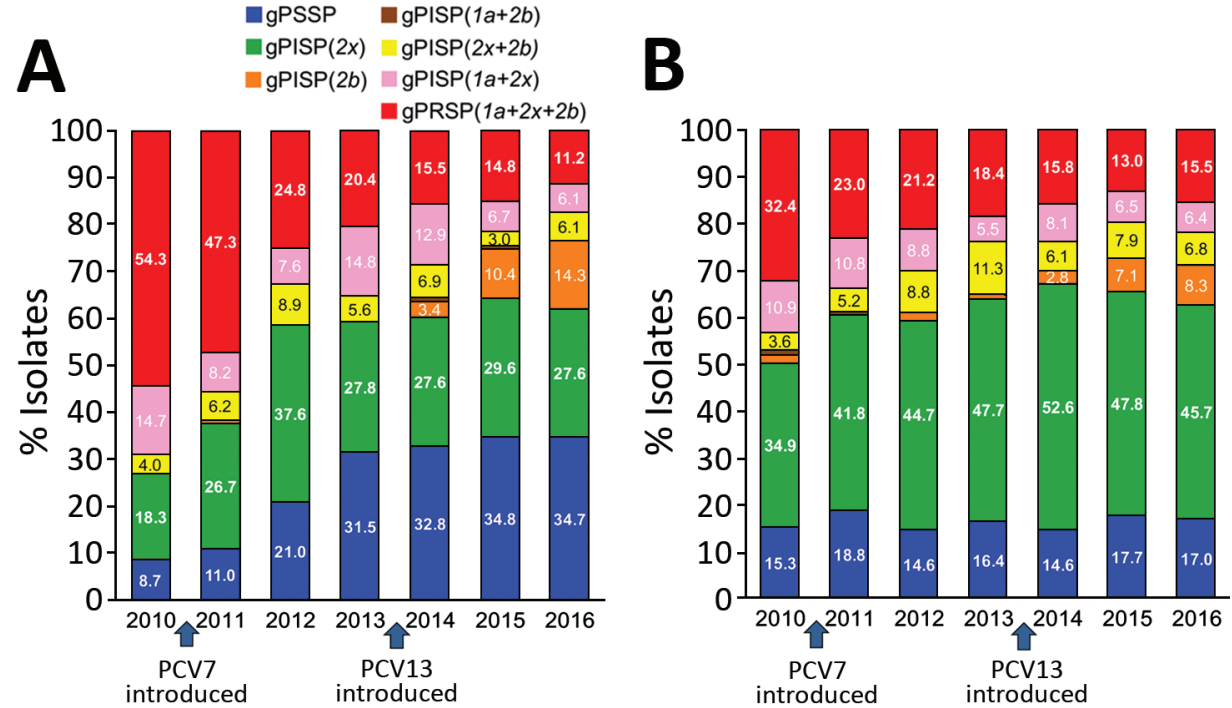

levofloxacin, accounted for $<1 \%$ of all isolates. These isolates showed no tendency to increase.

\section{Relationships between Serotypes and Resistance Genotypes}

Changes of serotypes and the penicillin resistance genotypes during the 3 periods (pre-PCV7, PCV7, and PCV13) are shown in online Technical Appendix Figures 2 (for children) and 3 (for adults). Decreases in gPRSP $(p b p 1 a+2 x+2 b)$ and gPISP $(p b p l a+2 x)$ were closely related to reduction of serotypes $6 \mathrm{~B}, 14,19 \mathrm{~F}, 23 \mathrm{~F}$, and $6 \mathrm{~A}$ in children and adults during the $\mathrm{PCV} 7$ period, and this link became stronger during the PCV13 period. Serotype 19A, including several gPRSP, decreased by half among children during the PCV13 period, but this change has not yet become evident among adults.

The proportions of PPSV23-nonPCV13 and NVT serotypes generally increased among children and adults during the PCV13 period. gPRSPs were newly identified in serotypes $15 \mathrm{~B}(\mathrm{n}=1), 15 \mathrm{C}(\mathrm{n}=1)$, and $16 \mathrm{~F}(\mathrm{n}=2)$ in isolates from children and in serotypes $6 \mathrm{C}(\mathrm{n}=2), 6 \mathrm{D}(\mathrm{n}=$ 2), $13(\mathrm{n}=1), 15 \mathrm{~B}(\mathrm{n}=1), 15 \mathrm{C}(\mathrm{n}=1), 16 \mathrm{~F}(\mathrm{n}=2), 23 \mathrm{~A}$ $(\mathrm{n}=1), 23 \mathrm{~B}(\mathrm{n}=1)$, and $34(\mathrm{n}=1)$ in isolates from adults.

Relationships between genotypic macrolide and penicillin resistances and serotypes are shown in online Technical Appendix Table 1. Strains possessing mef(A), erm(B), or both were identified in most of the serotypes, with the exception of serotypes $8,18 \mathrm{~B}, 28 \mathrm{~A}$, and 31 . No relationship was observed between macrolide resistance and penicillin resistance.

\section{Antimicrobial Susceptibility by Genotype}

Susceptibilities (50\% MIC, 90\% MIC, and MIC range) of 6 parenteral agents (penicillin, ampicillin, cefotaxime, meropenem, vancomycin, and levofloxacin) for
S. pneumoniae strains obtained from April 2014 through March 2017, corresponding to the PCV13 period ( $\mathrm{n}=$ 1,229), are shown in online Technical Appendix Table 2. Relationships between 6 genotypes for penicillin resistance and MICs of penicillin, ampicillin, cefotaxime, and meropenem for the strains are shown in online Technical Appendix Figure 4.

Because prevalence of gPRSP was reduced by the PCV vaccinations, the distribution of susceptibilities was shifted in favor of greater susceptibility, especially after introduction of PCV7. For penicillin and ampicillin, 90\% MICs were $2 \mu \mathrm{g} / \mathrm{mL}$; for cefotaxime, $1 \mu \mathrm{g} / \mathrm{mL}$; and for meropenem, $0.5 \mu \mathrm{g} / \mathrm{mL}$. gPRSP isolates showing high resistance for penicillin $(\geq 8 \mu \mathrm{g} / \mathrm{mL})$ were not found.

\section{STs by Serotypes and Resistance Genotypes}

STs by eBURST analyses for 2,849 pneumococcal strains are shown in Figure 3. These data are distinguished by ST and vaccine serotype (PCV7, PCV13-nonPCV7, PPSV23nonPCV13, and NVT). Details of relationships among clonal complexes (CCs), STs, serotypes, and resistance genotypes are listed in online Technical Appendix Table 3.

By MLST analysis, 273 different STs were identified. STs of gPRSP in 11 serotypes included in PPSV23-nonPCV13 and NVTs were noteworthy: 15B $(n=2)$, ST242 (belonging to CC242) and ST83 (derived from CC81); 6C $(\mathrm{n}=2)$, ST8352 (CC156) and ST5832 (CC5832); 6D ( $\mathrm{n}=$ 2), ST90 (CC156) and ST282 (CC81); $13(\mathrm{n}=1)$, ST10303 (CC156); 15A ( $=77)$, ST63, ST2105, ST2771, ST8354, and ST12000 (all CC63); 15C $(\mathrm{n}=2)$, ST83 and ST6138 $(\mathrm{CC} 81) ; 16 \mathrm{~F}(\mathrm{n}=4)$, ST8351 (CC3117); 23A $(\mathrm{n}=1)$, ST9619 (CC156); 23B ( $=1)$, ST2372 (CC156); $34(\mathrm{n}=$ 1), ST9395 (CC15); 35B ( $\mathrm{n}=55)$, ST558, ST1204, and 


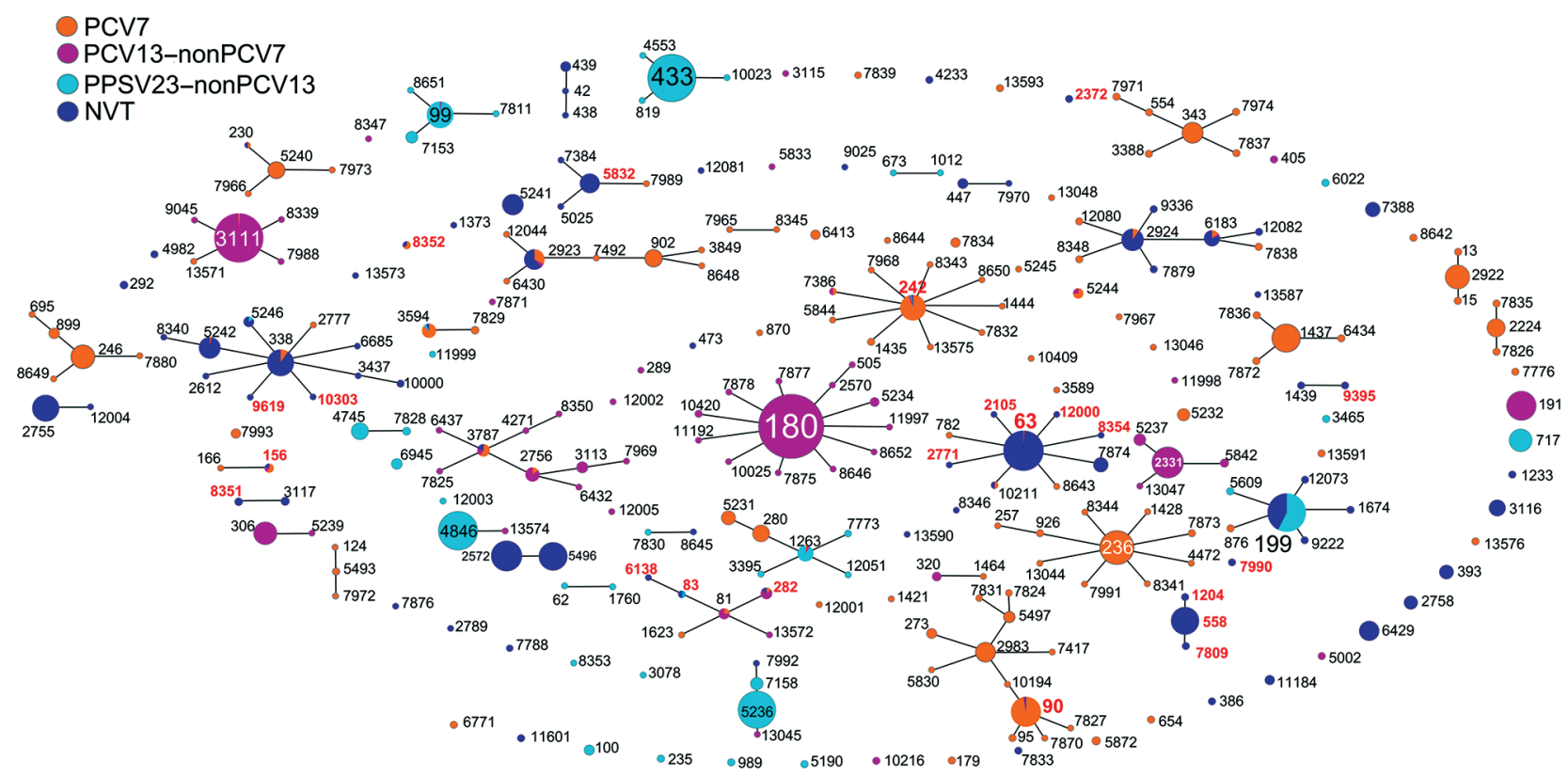

Figure 3. An eBURST (http://eburst.mlst.net/) diagram displaying pneumococcal sequence types (STs) causing invasive pneumococcal disease across patients of all age groups in Japan. All 2,849 strains are distinguished by colors to indicate PCV7, PCV13-nonPCV7, PPSV23-nonPCV13, and NVT. Size of each circle reflects the number of strains. ST numbers shown in red represent genotypes for penicillin-resistant Streptococcus pneumoniae confirmed among PPSV23-nonPCV13 and NVT as follows: 15B ( $n=2)$, ST242 and ST83; $6 C(n=2)$, ST8352 and ST5832; $6 D(n=2)$, ST90 and ST282; $13(n=1)$, ST10303; $15 A(n=77)$, ST63 $(n=73)$, ST2105, ST2771, ST8354, and ST12000; 15C $(n=2)$, ST83 and ST6138; 16F $(n=4)$, ST8351; 23A $(n=1)$, ST9619; 23B $(n=1)$, ST2372; $34(n=1)$, ST9395; 35B ( $n=55)$, ST558 ( $n=49)$, ST1204, ST7809, ST7990, and ST156. NVT, nonvaccine serotype; PCV7, 7-valent pneumococcal conjugate vaccine; PCV13, 13-valent pneumococcal conjugate vaccine; PPSV23, 23-valent pneumococcal polysaccharide vaccine.

ST7809 (all CC558); and the remaining ST156 (CC156) and ST7990 (singleton).

A total of 6 STs identified as gPRSP belonged to the large CC156 (Figure 4). STs of 3 serotypes (13, 23A, and 23B) were derived from ST338, which includes the Colombia $^{23 \mathrm{~F}-26}$ clone from the Pneumococcal Molecular Epidemiology Network (PMEN). Serotype 6C was derived from ST172, a neighbor of ST338. Of strains with serotypes 6D and $35 \mathrm{~B}$, each strain was distant from other gPRSPs. STs of serotypes $15 \mathrm{~B}, 15 \mathrm{C}$, and $6 \mathrm{D}$ among gPRSP belonged to CC81 (online Technical Appendix Figure 5).

In addition, STs of certain serotypes increasing in PPSV23-nonPCV13 and NVTs were noted. Serotype 12F was ST4846 (CC1527), 22F was ST433 (CC433), 23A included ST338 and ST5242 (CC156), and 24B/24F included ST2572 and ST5496 (CC2572).

\section{Discussion}

Wide use of PCVs among children in many countries has contributed to a dramatic reduction in incidence of IPD $(6,10,11,35-38)$, pneumonia $(12,13,39)$, and acute otitis media $(14,15)$ caused by $S$. pneumoniae, while providing indirect herd immunity benefits for adults $(11,23,40,41)$. Replacing PCV7 with PCV13 decidedly decreased serotype 19A isolates among causative pathogens, but in several countries, NVTs such as $15 \mathrm{~A}$ and $35 \mathrm{~B}$ increased. Gradual increases of NVTs, unfortunately, have blunted the effectiveness of conjugate vaccines (42).

In Japan, introduction of PCV7 in children $<5$ years of age began as an official government program in November 2010 , continuing until it was replaced with PCV13 in November 2013. PPSV23 vaccination for adults $\geq 65$ years of age was implemented in October 2014. We organized nationwide surveillance beginning in April 2010, with collection of pneumococcal strains from IPD patients in all age groups throughout Japan. In this article, we describe details of changes of serotypes, penicillin resistance genotypes, and MLST analyses that have followed implementation of PCV7 and PCV13 vaccination. As in other countries where PCV13 has been introduced, proportions of PCV13 serotypes among isolates from children and adults decreased significantly during the PCV13 period. In Japan, where population density is high, the decrease suggests early effectiveness of herd immunity not only among children but also among adults. However, serotypes 7F and 19A, included in PCV13, seem to be increasing among adults; for these serotypes, no indirect effect for adults is evident. These findings indicate a need for PCV13 vaccination of elderly 


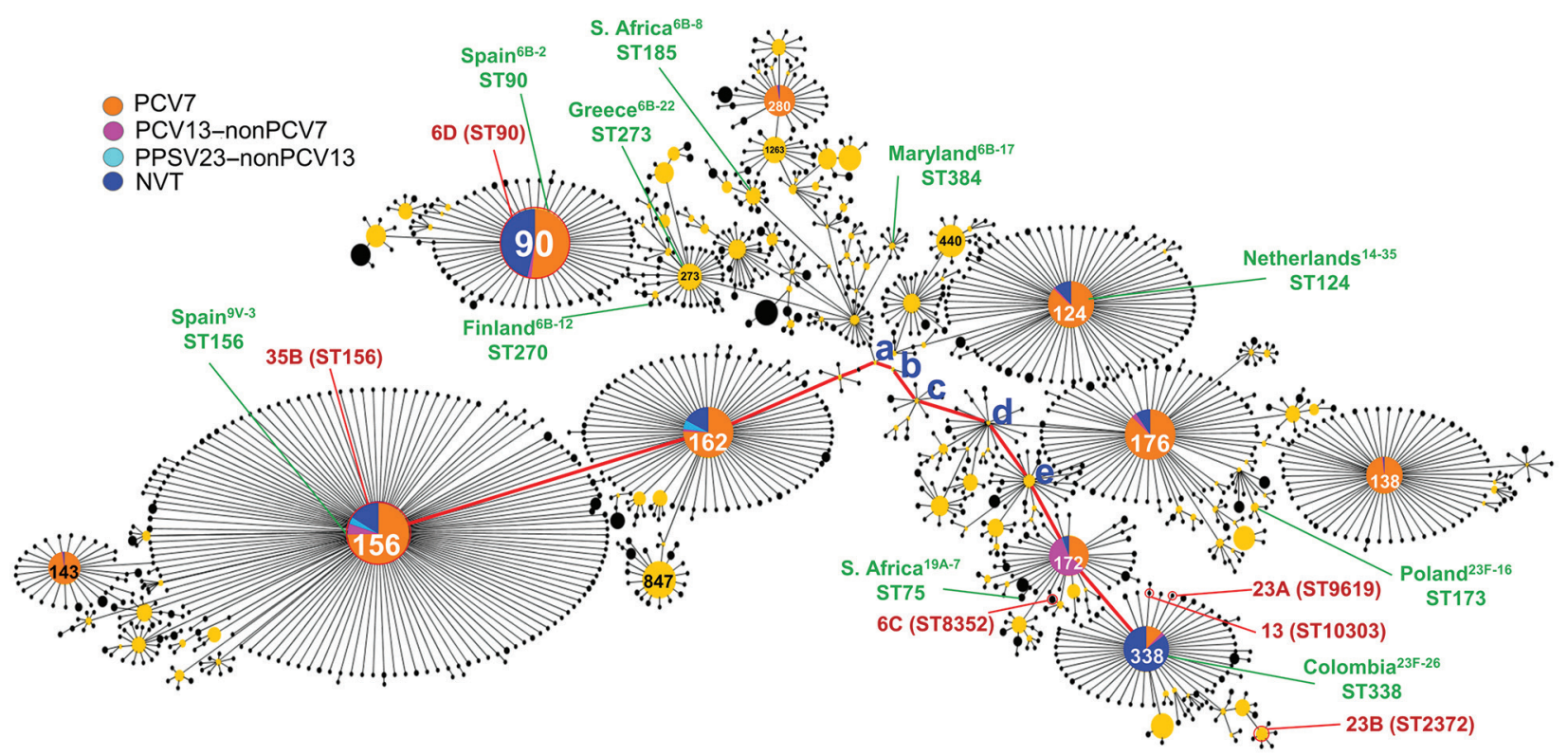

Figure 4. Details of Streptococcus pneumoniae clonal complex (CC) 156 ( $n=4,736$ ), including 1,308 sequence types obtained from the multilocus sequence typing website (https://pubmlst.org/spneumoniae/). Data include those from this study ( $n=359$ ). STs of 6 genotypes for penicillin-resistant S. pneumoniae identified in NVT serotypes belonged to CC156. STs of serotypes 6C, 13, 23A, and 23B were derived from ST338 and ST172 (shown in red). Serotypes 6D and 35B belonged to ST90 and ST156, respectively. The Pneumococcal Molecular Epidemiology Network clone identified in CC156 is also shown (in green). The red line indicates evolution from ST156 to ST338: a, ST8055; b, ST8618; c, ST4542; d, ST171; e, ST361. NVT, nonvaccine serotype; PCV7, 7-valent pneumococcal conjugate vaccine; PCV13, 13-valent pneumococcal conjugate vaccine; PPSV23, 23-valent pneumococcal polysaccharide vaccine; ST, sequence type.

and relatively immunocompromised persons, especially in Japan, where the population's average age is increasing. Of further concern is a test-negative design study conducted before introduction of PPSV23 to assess effectiveness of PPSV23 among elderly persons with community-acquired pneumonia in Japan. Effectiveness against communityacquired pneumonia caused by PPSV23 serotypes seemed low to moderate, depending on age group (43).

Proportions of many non-PCV13 serotypes during the PCV13 period have increased beyond proportions during the pre-PCV7 period. Nine serotypes $(10 \mathrm{~A}, 12 \mathrm{~F}, 15 \mathrm{~A}, 15 \mathrm{~B}$, $15 \mathrm{C}, 22 \mathrm{~F}, 24 \mathrm{~F}, 33 \mathrm{~F}$, and $35 \mathrm{~B}$ ) have increased significantly among children, and 5 serotypes $(12 \mathrm{~F}, 15 \mathrm{C}, 22 \mathrm{~F}, 23 \mathrm{~A}$, and 35B) have increased significantly among adults, showing considerable overlap between age groups. Among these serotypes, $15 \mathrm{~A}$ and $35 \mathrm{~B}$ have increased rapidly since PCV13 introduction in Japan, as has occurred in other countries $(18,25,28-30)$. The reason for increases in such serotypes is unclear; further epidemiologic surveillance may shed light on the matter.

Of note, gPRSP decreased sharply along with serotype replacements among children and adults. Highly penicillinresistant strains with MICs $\geq 8 \mu \mathrm{g} / \mathrm{mL}$, which sometimes were noted in serotypes $19 \mathrm{~F}$ and $23 \mathrm{~F}$ during the pre-PCV7 period (33), did not increase with introduction of PCVs.
Susceptibilities of most gPISP ( $p b p 2 b)$ in serotype 12F and of $\operatorname{gPISP}(p b p 2 x+2 b)$ in serotypes $23 \mathrm{~A}$ and $6 \mathrm{C}$ for penicillin and ampicillin ranged from 0.125 to $0.5 \mu \mathrm{g} / \mathrm{mL}$. Should mutation(s) occur in the regions encoding the conserved amino acids (STMK, SSN, and KTG) in the pbpla gene, antimicrobial selection pressure could easily favor development from gPISP to gPRSP.

One concern is the evolution of gPRSP among isolates from 11 NVTs according to MLST analysis. Most (all but 2) serotype $35 \mathrm{~B}$ isolates were found to belong to the same ST558 (CC558) that was reported from the United States in 1999 (44). Serotype 15A was identified as ST63, which belongs to CC63, as does the PMEN clone Sweden ${ }^{15 \mathrm{~A}-25}$. Each isolate of serotype 6D (ST282, CC81) and serotype 15B (ST83, CC81) was the same as those previously registered from South Korea (45) and Taiwan. These findings suggest that newly emerged resistant strains can spread rapidly between countries.

Among gPRSP identified in NVTs, STs of serotypes $6 \mathrm{C}, 13,23 \mathrm{~A}, 23 \mathrm{~B}$, and of both serotypes $6 \mathrm{D}$ and $35 \mathrm{~B}$, were noted to belong to $\mathrm{CC} 156$, which includes large numbers of isolates in ST156, ST90, ST162, ST124, ST176, and ST138; the PMEN clones Spain ${ }^{9 \mathrm{~V}-3}$, Netherlands ${ }^{14-35}$, Spain $^{6 \mathrm{~B}-2}$, Greece ${ }^{6 \mathrm{~B}-22}$, and S. Africa ${ }^{6 \mathrm{~B}-8}$ are representative among these. STs of serotypes $6 \mathrm{C}, 13,23 \mathrm{~A}$, and 23B were 
derived from ST172 and ST338, which diverged from ST171 (Figure 4, letter d) and evolved further. Serotypes of many isolates registered as ST172 or ST338 were either NVTs or one of the serotypes of PCV13-nonPCV7. These findings suggested that wide use of PCVs led to a decrease in STs belonging to PCV7 and PCV13 serotypes, but some STs detected among NVTs escaped from the vaccine pressure and are increasing, such as ST338. However, whether the new gPRSPs emerged in Japan or originated in another country is unknown.

Capsular switching in S. pneumoniae can occur as a result of homologous recombination at a site outside the cps locus. Of note, pbpla genes are located upstream and $p b p 2 x$ genes are located downstream of the cps locus (4648 ). Recombination including these $2 p b p$ genes, driven by antimicrobial pressure, can result in concomitant exchange of the cps locus. Such new ST strains arising from capsular switching can exhibit penicillin resistance and increase under antimicrobial selection pressure. The diversity of serotypes, resistant genotypes, and STs we describe reflects adaptability of $S$. pneumoniae to the human environment.

In conclusion, to assess whether gPRSP in NVTs will increase in the near future, sustained surveillance for IPD is needed. Control of pneumococcal infections, particularly in elderly and immunocompromised persons, requires development of further multivalent conjugate vaccines, new vaccines targeting a different microbial component, or both. Global consensus for appropriate use of antimicrobial drugs is also valuable for limiting spread of new resistant strains within and beyond national borders.

\section{Acknowledgments}

We thank Madoka Naitoh and Shinji Masuyoshi for their assistance. We are also grateful to the laboratory personnel and physicians who participated in this study.

Our study was funded in part by a grant to K.U. under the category "Research on Emerging and Re-emerging Infectious Diseases" (H22-013) from the Japanese Ministry of Health, Labour and Welfare.

\section{About the Author \\ Dr. Ubukata is a microbiologist at Keio University School of Medicine. Her research interests include molecular epidemiology, particularly with respect to respiratory infection, as well as comprehensive rapid identification of pathogens.}

\section{References}

1. O'Brien KL, Wolfson LJ, Watt JP, Henkle E, Deloria-Knoll M, McCall N, et al.; Hib and Pneumococcal Global Burden of Disease Study Team. Burden of disease caused by Streptococcus pneumoniae in children younger than 5 years: global estimates. Lancet. 2009;374:893-902. http://dx.doi.org/10.1016/ S0140-6736(09)61204-6
2. Whitney CG, Farley MM, Hadler J, Harrison LH, Bennett NM, Lynfield R, et al.; Active Bacterial Core Surveillance of the Emerging Infections Program Network. Decline in invasive pneumococcal disease after the introduction of proteinpolysaccharide conjugate vaccine. N Engl J Med. 2003;348:173746. http://dx.doi.org/10.1056/NEJMoa022823

3. Centers for Disease Control and Prevention. Direct and indirect effects of routine vaccination of children with 7-valent pneumococcal conjugate vaccine on incidence of invasive pneumococcal disease-United States, 1998-2003. MMWR Morb Mortal Wkly Rep. 2005;54:893-7.

4. Lexau CA, Lynfield R, Danila R, Pilishvili T, Facklam R, Farley MM, et al.; Active Bacterial Core Surveillance Team. Changing epidemiology of invasive pneumococcal disease among older adults in the era of pediatric pneumococcal conjugate vaccine. JAMA. 2005;294:2043-51. http://dx.doi.org/10.1001/ jama.294.16.2043

5. Hsu HE, Shutt KA, Moore MR, Beall BW, Bennett NM, Craig AS, et al. Effect of pneumococcal conjugate vaccine on pneumococcal meningitis. N Engl J Med. 2009;360:244-56. http://dx.doi.org/ 10.1056/NEJMoa0800836

6. Pilishvili T, Lexau C, Farley MM, Hadler J, Harrison LH, Bennett NM, et al.; Active Bacterial Core Surveillance/Emerging Infections Program Network. Sustained reductions in invasive pneumococcal disease in the era of conjugate vaccine. J Infect Dis. 2010;201:32-41. http://dx.doi.org/10.1086/648593

7. Hicks LA, Harrison LH, Flannery B, Hadler JL, Schaffner W, Craig AS, et al. Incidence of pneumococcal disease due to non-pneumococcal conjugate vaccine (PCV7) serotypes in the United States during the era of widespread PCV7 vaccination, 1998-2004. J Infect Dis. 2007;196:1346-54. http://dx.doi.org/ $10.1086 / 521626$

8. Moore MR, Gertz RE Jr, Woodbury RL, Barkocy-Gallagher GA, Schaffner W, Lexau C, et al. Population snapshot of emergent Streptococcus pneumoniae serotype 19A in the United States, 2005. J Infect Dis. 2008;197:1016-27. http://dx.doi.org/10.1086/528996

9. Centers for Disease Control and Prevention. Licensure of a 13-valent pneumococcal conjugate vaccine (PCV13) and recommendations for use among children-Advisory Committee on Immunization Practices (ACIP), 2010. MMWR Morb Mortal Wkly Rep. 2010;59:258-61.

10. Kaplan SL, Barson WJ, Lin PL, Romero JR, Bradley JS, Tan TQ, et al. Early trends for invasive pneumococcal infections in children after the introduction of the 13-valent pneumococcal conjugate vaccine. Pediatr Infect Dis J. 2013;32:203-7. http://dx.doi.org/10.1097/INF.0b013e318275614b

11. Moore MR, Link-Gelles R, Schaffner W, Lynfield R, Lexau C, Bennett NM, et al. Effect of use of 13-valent pneumococcal conjugate vaccine in children on invasive pneumococcal disease in children and adults in the USA: analysis of multisite, population-based surveillance. Lancet Infect Dis. 2015;15:301-9. http://dx.doi.org/10.1016/S1473-3099(14)71081-3

12. Griffin MR, Zhu Y, Moore MR, Whitney CG, Grijalva CG. U.S. hospitalizations for pneumonia after a decade of pneumococcal vaccination. N Engl J Med. 2013;369:155-63. http://dx.doi.org/ 10.1056/NEJMoa1209165

13. Angoulvant F, Levy C, Grimprel E, Varon E, Lorrot M, Biscardi S, et al. Early impact of 13-valent pneumococcal conjugate vaccine on community-acquired pneumonia in children. Clin Infect Dis. 2014;58:918-24. http://dx.doi.org/10.1093/cid/ciu006

14. Ben-Shimol S, Givon-Lavi N, Leibovitz E, Raiz S, Greenberg D, Dagan R. Near-elimination of otitis media caused by 13-valent pneumococcal conjugate vaccine (PCV) serotypes in southern Israel shortly after sequential introduction of 7-valent/13-valent PCV. Clin Infect Dis. 2014;59:1724-32. http://dx.doi.org/10.1093/ $\mathrm{cid} / \mathrm{ciu} 683$ 
15. Kaplan SL, Center KJ, Barson WJ, Ling-Lin P, Romero JR, Bradley JS, et al. Multicenter surveillance of Streptococcus pneumoniae isolates from middle ear and mastoid cultures in the 13-valent pneumococcal conjugate vaccine era. Clin Infect Dis. 2015;60:1339-45

16. Kempf M, Varon E, Lepoutre A, Gravet A, Baraduc R, Brun M, et al. Decline in antibiotic resistance and changes in the serotype distribution of Streptococcus pneumoniae isolates from children with acute otitis media; a 2001-2011 survey by the French Pneumococcal Network. Clin Microbiol Infect. 2015;21:35-42. http://dx.doi.org/10.1016/j.cmi.2014.08.009

17. Williams WW, Lu PJ, O'Halloran A, Kim DK, Grohskopf LA, Pilishvili T, et al. Surveillance of vaccination coverage among adult populations - United States, 2015. MMWR Surveill Summ. 2017;66:1-28. http://dx.doi.org/10.15585/mmwr.ss6611a1

18. Steens A, Bergsaker MA, Aaberge IS, Rønning K, Vestrheim DF. Prompt effect of replacing the 7-valent pneumococcal conjugate vaccine with the 13-valent vaccine on the epidemiology of invasive pneumococcal disease in Norway. Vaccine. 2013;31:6232-8. http://dx.doi.org/10.1016/j.vaccine.2013.10.032

19. Moore CE, Paul J, Foster D, Mahar SA, Griffiths D, Knox K, et al.; Oxford Invasive Pneumococcal Surveillance Group. Reduction of invasive pneumococcal disease 3 years after the introduction of the 13 -valent conjugate vaccine in the Oxfordshire region of England. J Infect Dis. 2014;210:1001-11. http://dx.doi.org/10.1093/infdis/ jiu 213

20. Harboe ZB, Dalby T, Weinberger DM, Benfield T, Mølbak K, Slotved HC, et al. Impact of 13-valent pneumococcal conjugate vaccination in invasive pneumococcal disease incidence and mortality. Clin Infect Dis. 2014;59:1066-73. http://dx.doi.org/ 10.1093/cid/ciu524

21. Waight PA, Andrews NJ, Ladhani SN, Sheppard CL, Slack MP, Miller E. Effect of the 13-valent pneumococcal conjugate vaccine on invasive pneumococcal disease in England and Wales 4 years after its introduction: an observational cohort study. Lancet Infect Dis. 2015;15:535-43. http://dx.doi.org/10.1016/ S1473-3099(15)70044-7

22. Alari A, Chaussade H, Domenech De Cellès M, Le Fouler L, Varon E, Opatowski L, et al. Impact of pneumococcal conjugate vaccines on pneumococcal meningitis cases in France between 2001 and 2014: a time series analysis. BMC Med. 2016;14:211. http://dx.doi.org/10.1186/s12916-016-0755-7

23. Hays C, Vermee Q, Agathine A, Dupuis A, Varon E, Poyart C, et al.; the ORP Ile de France Ouest. Demonstration of the herd effect in adults after the implementation of pneumococcal vaccination with PCV13 in children. Eur J Clin Microbiol Infect Dis. 2017;36:831-8. http://dx.doi.org/10.1007/s10096-016-2868-5

24. Gertz RE Jr, Li Z, Pimenta FC, Jackson D, Juni BA, Lynfield R, et al.; Active Bacterial Core Surveillance Team. Increased penicillin nonsusceptibility of nonvaccine-serotype invasive pneumococci other than serotypes 19A and 6A in post-7-valent conjugate vaccine era. J Infect Dis. 2010;201:770-5. http://dx.doi.org/10.1086/650496

25. Richter SS, Heilmann KP, Dohrn CL, Riahi F, Diekema DJ, Doern GV. Pneumococcal serotypes before and after introduction of conjugate vaccines, United States, 1999-2011. Emerg Infect Dis. 2013;19:1074-83. http://dx.doi.org/10.3201/eid1907.121830

26. Mendes RE, Costello AJ, Jacobs MR, Biek D, Critchley IA, Jones RN. Serotype distribution and antimicrobial susceptibility of USA Streptococcus pneumoniae isolates collected prior to and post introduction of 13-valent pneumococcal conjugate vaccine. Diagn Microbiol Infect Dis. 2014;80:19-25. http://dx.doi.org/10.1016/ j.diagmicrobio.2014.05.020

27. Richter SS, Musher DM. The ongoing genetic adaptation of Streptococcus pneumoniae. J Clin Microbiol. 2017;55:681-5. http://dx.doi.org/10.1128/JCM.02283-16
28. Olarte L, Kaplan SL, Barson WJ, Romero JR, Lin PL, Tan TQ, et al. Emergence of multidrug-resistant pneumococcal serotype 35B among children in the United States. J Clin Microbiol. 2017;55:724-34. http://dx.doi.org/10.1128/JCM.01778-16

29. van der Linden M, Perniciaro S, Imöhl M. Increase of serotypes $15 \mathrm{~A}$ and $23 \mathrm{~B}$ in IPD in Germany in the PCV13 vaccination era. BMC Infect Dis. 2015;15:207. http://dx.doi.org/10.1186/ s12879-015-0941-9

30. Janoir C, Lepoutre A, Gutmann L, Varon E. Insight into resistance phenotypes of emergent non 13-valent pneumococcal conjugate vaccine type pneumococci isolated from invasive disease after 13-valent pneumococcal conjugate vaccine implementation in France. Open Forum Infect Dis. 2016;3:ofw020. http://dx.doi.org/ 10.1093/ofid/ofw020

31. Chiba N, Morozumi M, Shouji M, Wajima T, Iwata S, Ubukata K; Invasive Pneumococcal Diseases Surveillance Study Group. Changes in capsule and drug resistance of pneumococci after introduction of PCV7, Japan, 2010-2013. Emerg Infect Dis. 2014;20:1132-9. http://dx.doi.org/10.3201/eid2007.131485

32. Ubukata K, Chiba N, Hanada S, Morozumi M, Wajima T, Shouji M, et al.; Invasive Pneumococcal Diseases Surveillance Study Group. Serotype changes and drug resistance in invasive pneumococcal diseases in adults after vaccinations in children, Japan, 2010-2013. Emerg Infect Dis. 2015;21:1956-65. http://dx.doi.org/10.3201/eid2111.142029

33. Chiba N, Morozumi M, Ubukata K. Application of the real-time PCR method for genotypic identification of $\beta$-lactam resistance in isolates from invasive pneumococcal diseases. Microb Drug Resist. 2012;18:149-56. http://dx.doi.org/10.1089/mdr.2011.0102

34. Clinical and Laboratory Standards Institute. Performance standards for antimicrobial susceptibility testing; 25th informational supplement (M100-S25). Wayne (PA): The Institute; 2015.

35. Miller E, Andrews NJ, Waight PA, Slack MP, George RC. Herd immunity and serotype replacement 4 years after sevenvalent pneumococcal conjugate vaccination in England and Wales: an observational cohort study. Lancet Infect Dis. 2011;11:760-8. http://dx.doi.org/10.1016/S1473-3099(11)70090-1

36. Weinberger DM, Malley R, Lipsitch M. Serotype replacement in disease after pneumococcal vaccination. Lancet. 2011;378: 1962-73. http://dx.doi.org/10.1016/S0140-6736(10)62225-8

37. Lepoutre A, Varon E, Georges S, Dorléans F, Janoir C, Gutmann L, et al.; Microbiologists of Epibac; ORP Networks. Impact of the pneumococcal conjugate vaccines on invasive pneumococcal disease in France, 2001-2012. Vaccine. 2015;33:359-66. http://dx.doi.org/10.1016/j.vaccine.2014.11.011

38. Gaviria-Agudelo CL, Jordan-Villegas A, Garcia C, McCracken GH Jr. The effect of 13-valent pneumococcal conjugate vaccine on the serotype distribution and antibiotic resistance profiles in children with invasive pneumococcal disease. J Pediatric Infect Dis Soc. 2017;6:253-9. http://dx.doi.org/10.1093/jpids/piw005

39. Olarte L, Barson WJ, Barson RM, Romero JR, Bradley JS, Tan TQ, et al. Pneumococcal pneumonia requiring hospitalization in US children in the 13-valent pneumococcal conjugate vaccine era. Clin Infect Dis. 2017;64:1699-704. http://dx.doi.org/10.1093/cid/cix115

40. Pilishvili T, Bennett NM. Pneumococcal disease prevention among adults: strategies for the use of pneumococcal vaccines. Vaccine. 2015;33(Suppl 4):D60-5. http://dx.doi.org/10.1016/ j.vaccine.2015.05.102

41. van der Linden M, Falkenhorst G, Perniciaro S, Imöhl M. Effects of infant pneumococcal conjugate vaccination on serotype distribution in invasive pneumococcal disease among children and adults in Germany. PLoS One. 2015;10:e0131494. http://dx.doi.org/10.1371/ journal.pone. 0131494

42. Weinberger R, von Kries R, van der Linden M, Rieck T, Siedler A, Falkenhorst G. Invasive pneumococcal disease in children under 16 years of age: incomplete rebound in incidence 
after the maximum effect of PCV13 in 2012/13 in Germany. Vaccine. 2018;36:572-7. http://dx.doi.org/10.1016/j.vaccine.2017.11.085

43. Suzuki M, Dhoubhadel BG, Ishifuji T, Yasunami M, Yaegashi M, Asoh N, et al.; Adult Pneumonia Study Group-Japan. Serotype-specific effectiveness of 23-valent pneumococcal polysaccharide vaccine against pneumococcal pneumonia in adults aged 65 years or older: a multicentre, prospective, test-negative design study. Lancet Infect Dis. 2017;17:313-21. http://dx.doi.org/10.1016/S1473-3099(17)30049-X

44. Beall B, McEllistrem MC, Gertz RE Jr, Boxrud DJ, Besser JM, Harrison LH, et al.; Active Bacterial Core Surveillance/Emerging Infections Program Network. Emergence of a novel penicillinnonsusceptible, invasive serotype 35B clone of Streptococcus pneumoniae within the United States. J Infect Dis. 2002;186: 118-22. http://dx.doi.org/10.1086/341072

45. Ko KS, Baek JY, Song JH. Multidrug-resistant Streptococcus pneumoniae serotype 6D clones in South Korea. J Clin Microbiol. 2012;50:818-22. http://dx.doi.org/10.1128/JCM.05895-11
46. Brueggemann AB, Pai R, Crook DW, Beall B. Vaccine escape recombinants emerge after pneumococcal vaccination in the United States. PLoS Pathog. 2007;3:e168. http://dx.doi.org/ 10.1371/journal.ppat.0030168

47. Wyres KL, Lambertsen LM, Croucher NJ, McGee L, von Gottberg A, Liñares J, et al. Pneumococcal capsular switching: a historical perspective. J Infect Dis. 2013;207:439-49. http://dx.doi.org/10.1093/infdis/jis703

48. Chiba N, Murayama SY, Morozumi M, Iwata S, Ubukata K. Genome evolution to penicillin resistance in serotype 3 Streptococcus pneumoniae by capsular switching. Antimicrob Agents Chemother. 2017;61:e00478-17. http://dx.doi.org/10.1128/ AAC.00478-17

Address for correspondence: Kimiko Ubukata, Keio University School of Medicine, Department of Infectious Diseases, 35 Shinanomachi Shinjyuku-ku, Tokyo 160-8582, Japan; email: ubukatak@keio.jp

\section{May 2017: Antimicrobial Resistance}

- Exposure Characteristics of Hantavirus Pulmonary Syndrome Patients, United States, 1993-2015

- Increased Neurotropic Threat from Burkholderia pseudomallei Strains with a B. mallei-like Variation in the bimA Motility Gene, Australia

- Population Genomics of Legionella longbeachae and Hidden Complexities of Infection Source Attribution

- Prevention of Chronic Hepatitis B after 3 Decades of Escalating Vaccination Policy, China

- Lack of Durable Cross-Neutralizing Antibodies against Zika Virus from Dengue Virus Infection

- Use of Blood Donor Screening Data to Estimate Zika Virus Incidence, Puerto Rico, April-August 2016
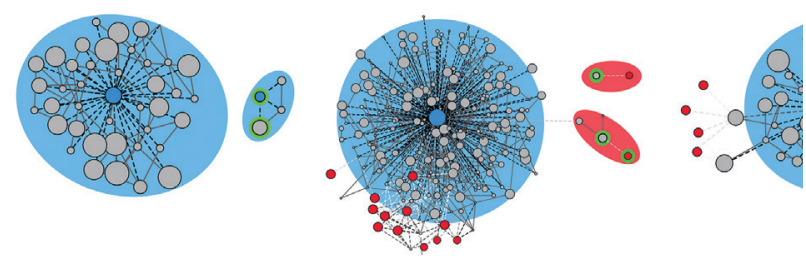

- Invasive Nontuberculous Mycobacterial Infections among Cardiothoracic Surgical Patients Exposed to Heater-Cooler Devices

- Anthrax Cases Associated with Animal-Hair Shaving Brushes

- Increasing Macrolide and Fluoroquinolone Resistance in Mycoplasma genitalium

- Survey of Treponemal Infections in Free-Ranging and Captive Macaques, 1999-2012

- Estimated Incubation Period for Zika Virus Disease



- Population Responses during the Pandemic Phase of the Influenza $\mathrm{A}(\mathrm{H} 1 \mathrm{~N} 1)$ pdm09 Epidemic, Hong Kong, China

- Phenotypic and Genotypic Shifts in Hepatitis B Virus in Treatment-Naive Patients, Taiwan, 2008-2012

- No Such Thing as Chronic Q Fever

- Reassortant Clade 2.3.4.4 Avian Influenza A(H5N6) Virus in a Wild Mandarin Duck, South Korea, 2016

- Amoxicillin and Ceftriaxone as Treatment Alternatives to Penicillin for Maternal Syphilis

- Azithromycin Resistance and Decreased Ceftiraxone Susceptibility in Neisseria gonorrhoeae, Hawaii, USA

- Regional Transmission of Salmonella Paratyphi A, China, 1998-2012

- Exposure Risk for Infection and Lack of Human-toHuman Transmission of Mycobacterium ulcerans Disease, Australia

- Virulence Analysis of Bacillus cereus Isolated after Death of Preterm Neonates, Nice, France

- The Discovery of Penicillin-New Insights after More than 75 years of Clinical Use

- Persistence of Zika Virus in Breast Milk after Infection in Late Stage of Pregnancy

\section{EMERGING INFECTIOUS DISEASES

